Voors, A. A. et al. (2016) A systems BIOlogy Study to TAilored treatment in chronic heart failure: rationale, design, and baseline characteristics of BIOSTATCHF. European Journal of Heart Failure, 18(6), pp. 716-726.

There may be differences between this version and the published version. You are advised to consult the publisher's version if you wish to cite from it.

This is the peer reviewed version of the following article: Voors, A. A. et al. (2016) A systems BIOlogy Study to TAilored treatment in chronic heart failure: rationale, design, and baseline characteristics of BIOSTAT-CHF. European Journal of Heart Failure, 18(6), pp. 716-726, which has been published in final form at http://dx.doi.org/10.1002/ejhf.531. This article may be used for non-commercial purposes in accordance with Wiley Terms and Conditions for Self-Archiving.

http://eprints.gla.ac.uk/128157/

Deposited on: 21 November 2016

Enlighten - Research publications by members of the University of Glasgow http://eprints.gla.ac.uk 


\title{
A systems BIOlogy Study to TAilored Treatment in Chronic Heart Failure: Rationale, design, and baseline characteristics of BIOSTAT-CHF
}

\begin{abstract}
A.A. Voors, MD (1), S.D. Anker, MD (2), J.G. Cleland, MD (3), K. Dickstein, MD (4), G. Filippatos, MD (5), P. van der Harst, MD (1), H.L. Hillege, MD (1), C.C. Lang, MD (6), J.M. ter Maaten, MD (1) L. Ng, MD (7), P. Ponikowski, MD (8) N.J Samani, MD (7), D.J. van Veldhuisen, MD (1), F. Zannad, MD (9), A.H. Zwinderman, MD (10), M. Metra, MD (11)
\end{abstract}

1. Department of Cardiology, University of Groningen, Groningen, the Netherlands

2. Innovative Clinical Trials, Department of Cardiology \& Pneumology, University Medical Center Göttingen (UMG), Göttingen, Germany

3. National Heart \& Lung Institute, Royal Brompton \& Harefield Hospitals, Imperial College, London, United Kingdom.

4. University of Bergen, Stavanger University Hospital, Stavanger, Norway

5. Department of Cardiology, Heart Failure Unit, Athens University Hospital Attikon, Athens, Greece

6. School of Medicine Centre for Cardiovascular and Lung Biology, Division of Medical Sciences, University of Dundee, Ninewells Hospital \& Medical School, Dundee, UK

7. Department of Cardiovascular Sciences, University of Leicester, Glenfield Hospital, Leicester, UK and NIHR Leicester Cardiovascular Biomedical Research Unit, Glenfield Hospital, Leicester, LE3 9QP, UK

8. Department of Heart Diseases, Wroclaw Medical University, Poland and Cardiology Department, Military Hospital, Wroclaw, Poland.

9. Henri Poincare University of Nancy, Nancy, France

10. Department of Epidemiology, Academic Medical Center, Amsterdam, The Netherlands

11. Institute of Cardiology, Department of medical and surgical specialties, radiological sciences and public health; University of Brescia, Italy 
Address for Correspondence: A.A. Voors, MD. Department of Cardiology, University Medical Center

Groningen, Hanzeplein 1, 9713 GZ Groningen, the Netherlands. Tel +31503612355; Fax +31503614391;

e-mail: a.a.voors@umcg.nl 


\section{Abstract}

Despite major improvements in pharmacological and device treatments, heart failure remains a syndrome with a high morbidity and mortality, a poor quality of life, and high health care costs. Given extensive heterogeneity among patients with heart failure, substantial differences in the response to therapy can be expected. We hypothesize that individualized therapy is an essential next step to improve outcomes in patients with heart failure. BIOSTAT-CHF uses a novel systems biology approach to develop a model that predicts response to therapy, incorporating demographics, biomarkers, genomewide analysis, and proteomics. For this purpose, we included 2516 patients with worsening signs and/or symptoms of heart failure from 11 European countries, who were considered to be on suboptimal medical treatment. Another 1738 patients from Scotland were included in a validation cohort. Overall, both patient cohorts were well matched. The majority of patients were hospitalised for acute heart failure, and the remainder presented with worsening signs and/or symptoms of heart failure at the outpatient clinic. Approximately half of the patients in NYHA class III and 10-15\% of patients had HFpEF. According to study design, all patients used diuretics, but due to the inclusion criteria of both cohorts, patients were not on optimal, evidence-based medical therapy: 70/72\% used an ACEinhibitor/Angiotensin Receptor Blocker, and < 50\% used the optimal dose. Beta-blockers were used in $73 / 83 \%$ of patients and only $32 / 46 \%$ used the optimal dose. The predictive models for response to therapy are required to evaluate and test alternative therapies for patients with a suboptimal response and thus improve care for patients with heart failure. BIOSTAT-CHF was funded by a grant from the European Commission (FP7-242209-BIOSTAT-CHF; EudraCT 2010-020808-29) 


\section{Introduction}

Despite advances in the treatment of patients with heart failure, the prognosis of patients with worsening symptoms of heart failure remains poor with up to $40 \%$ being dead or readmitted to hospital within 1 year. (1-4) In a recent European Survey in patients with chronic stable heart failure, more than $17 \%$ of patients died or were hospitalized for heart failure within a year.(5) Prognosis is even worse in patients admitted for acute heart failure. In the same registry, one year mortality or heart failure hospitalization in patients admitted for acute heart failure was more than 35\%. (5) Similar mortality rates have been shown in other registries, which also showed that $30-40 \%$ of patients were rehospitalized within 1 year after hospital admission.(2) Thus, although the prognosis of heart failure patients has improved, mortality and rehospitalisation remain high, especially in patients with worsening signs and/or symptoms of heart failure.

There are several explanations for the persistent high mortality and morbidity in patients with worsening signs and symptoms of heart failure. First, patients are often not optimally treated due to compliance of patients and health care providers. Second, better treatments might be needed to further improve clinical outcome. However, it is generally observed that the absolute effect of new pharmacological agents has declined over the last 2 decades.(6) In addition, from 2004- 2010, several large drug trials in patients with heart failure failed to show any benefit.(7-9) This may reflect the increased difficulty to further reduce mortality and morbidity, on top off effective drugs, the so-called a law of diminishing returns.

We propose that future significant improvements can be achieved when therapy is individualized and tailored to the patient's profile. Given the large heterogeneity of patients with heart failure with multiple aetiologies, cardiac phenotypes and co-morbidities, a currently advocated one-size-fits-all approach is not appropriate in every patient. Large randomized clinical trials might show an overall beneficial effect, but treatment may not necessarily be beneficial in every patient subgroup. A successful trial just means that significantly more people benefitted. Many patients will never experience the endpoints captured and treatment may have made no difference to their outcome. The residual high mortality and morbidity reflect groups of patients who do not benefit from existing therapies. 


\section{Main objectives of BIOSTAT-CHF}

The main aim of BIOSTAT-CHF is to characterize biological pathways related to response/no-response to guideline-recommended pharmacological therapy for heart failure. Patients will be profiled at several levels, including clinical characteristics, genetic background, and protein expression. Using a systems biology approach, these levels will be integrated to search for pathways which may explain the individual patient response to therapy.

\section{Design of BIOSTAT-CHF}

The design of the BIOSTAT-CHF project is presented in figure 1. In brief, from an index cohort of 2516 patients (Work Package 2 and 6), information was collected to create a risk score for non-response to therapy defined as death or heart failure hospitalization. This information includes blood and urinary biomarkers (Work Package 3), proteomics (Work Package 4) and genomics (Work Package 5). The score will be made using a systems biology approach (Work Package 7). The score will then be validated in a second cohort of 1738 patients (Work Package 8).

\section{Index cohort}

\section{Study design}

This was a multicenter, multinational, prospective, observational study. The study population consisted of 2516 patients from 69 centres in 11 European countries. For the list of centres and the investigators, see appendix 1. The recruitment period was 24 months, starting from December 2010. The last patient was included on December 15, 2012. Median follow-up was 21 months [interquartile range 15-27 months] .

Study population

- Patients were aged $\geq 18$ years with symptoms of new-onset or worsening heart failure 
- They had objective evidence of cardiac dysfunction documented either by

o left ventricular ejection fraction of $\leq 40 \%$ OR

o plasma concentrations of BNP and/or NT-proBNP $>400 \mathrm{pg} / \mathrm{ml}$ or $>2,000 \mathrm{pg} / \mathrm{ml}$, respectively

- They were treated with either oral or intravenous furosemide $\geq 40 \mathrm{mg} /$ day or equivalent at the time of inclusion

- Not previously treated with evidence based therapies (ACEi/ARBs and beta-blockers) or receiving $\leq 50 \%$ of the target doses of these drugs at the time of inclusion

- Were anticipated to be initiated or up-titrated with ACEi/ARBs and/or beta-blocker therapy by the treating physician

Patients could be enrolled as in-patients or from out-patient clinics.

- Exclusion criteria were the following: known diagnosis of septicaemia, known diagnosis of acute myocarditis or hypertrophic obstructive, restrictive, or constrictive cardiomyopathy, heart transplant recipient or admitted for cardiac transplantation or LVAD surgery, anticipated need of surgery or any cardiovascular intervention, except implantable cardioverter defibrillator and/or cardiac resynchronization therapy, within 4 weeks, current known inability to follow instructions or comply with follow-up procedures, treatment with medications or devices not approved in Europe. Patients with concomitant pulmonary disease, even if severe, valvular disease, acute coronary syndrome or stroke, could be included when the primary diagnosis for admission to hospital or outpatient clinic visit was heart failure, rather than the concomitant condition.

\section{Main study endpoints}

All deaths and hospitalizations were recorded. The primary outcome of interest was time to a composite of death or unscheduled hospitalizations for heart failure. The heart failure hospitalizations were determined by the investigator, and were not adjudicated by a central adjudication committee.

Study Plan 
Eligible patients signed informed consent and were included in the study. Medical history, current use of medication, and a physical examination were recorded at baseline. All patients underwent a 6-minute walk test and completed EQ5D and KCCQ questionnaires. Plasma and serum (total of $80 \mathrm{cc}$ ) and urine were sampled for analysis. Performance of standard echocardiography was strongly recommended but not mandatory for inclusion into the study. The study recorded changes in the use of medication. During the first three months the investigators were required to optimize treatment of heart failure, including the initiation of evidence based therapies and the up titration of current therapies to the doses indicated in the European Society of Cardiology 2008 guidelines.(10) The subsequent 6 months were considered as the maintenance phase and no further treatment optimization was predicted, except in case of changes in the clinical status. At 9 months, a second study-mandated visit was performed. Current symptoms and medication were recorded, a physical examination, 6-minute walk test, and EQ5D and KCCQ questionnaires were completed and more blood was collected. Patients were subsequently followed, by standard clinic follow-up or telephone contact after intervals of 6 months, until the end of follow-up on April 1, 2015.

\section{Validation cohort}

\section{Study design}

The BIOSTAT-CHF validation cohort was designed as a multicenter, prospective, observational study. The study population consisted of 1738 patients from 6 centres in Scotland, UK. For the list of centers and the investigators, see appendix 2. The recruitment period started in October 2010 and was completed in April 2014. Median follow-up was 21 months [interquartile range 11-32 months].

\section{Study population}

- Patients were aged $\geq 18$ years with a diagnosis of heart failure based on

- Echocardiographic evidence of left ventricular systolic dysfunction

O A previous documented admission with heart failure requiring diuretics

- Were on treatment with furosemide $\geq 20 \mathrm{mg} /$ day or equivalent 
- Were not previously treated or receiving $\leq 50 \%$ of target doses of ACE inhibitors/ARBs and/or beta-blockers according to the European Society of Cardiology 2008 guidelines.

- There was an anticipated initiation or up-titration of an ACEi/ARB and/or a beta blocker

- Patients could be enrolled as in-patients or from out-patient clinics.

Main study endpoints

All deaths and hospitalizations were recorded. The primary outcome of interest was time to a composite of all-cause death or unscheduled hospitalizations for heart failure during follow-up. The heart failure hospitalizations were determined by the investigator, and were not adjudicated by a central adjudication committee.

\section{Study Plan}

Following written Informed Consent the following assessments were performed: demographics (including medication), EQ5D and KCCQ Quality of Life Questionnaires were issued for completion by the patient, a medical history and physical examination, and blood draw of approximately 80 cc of plasma and serum, a urine specimen and a 12-lead resting ECG. If not already performed, an echocardiogram was performed by the clinical research fellow.

\section{Biomarkers}

Biomarkers from several pathophysiological domains were measured, including markers of inflammation, apoptosis, remodelling, myocyte stress/injury, angiogenesis, endothelial function, and several markers of renal function. For an overview of all novel and traditional biomarkers available in BIOSTAT-CHF, please see table 2.

\section{Proteomics}


Proteomics and peptidomics were performed using high resolution mass spectrometry (MS) to enable highly accurate mass determination of ions to study the proteome of plasma, using two soft ionisation techniques, MALDI (matrix assisted laser desorption and ionisation MS) for peptidomics and ESI (electrospray ionisation) for bottom-up proteomics using two-dimensional liquid chromatography highdefinition MS (2D LC HDMS, with ion mobility spectroscopy). A series of informatics software packages were used to identify and quantify proteins from the highly complex data generated from the 2D LC HDMS. Machine learning algorithms employing support vector machines, neural networks or decision forests as the core classifier were employed on the high-dimensional peptidomic data, to seek predictive models for responder status. In addition, evolutionary search methods (genetic algorithms) were utilised to reduce the peptidomic feature set to those that are most effective at classification. Detailed bioinformatic analyses of the 2D LC HDMS data were performed on a subset of the patient cases showing good or poor outcomes, in order to formulate the optimal set of predictive protein features. These candidate proteins were verified using immunoassay or selected reaction monitoring. From this, a set of immunoassays was devised for the best 10 candidate proteomic biomarkers to be tested on the index and validation cohort.

\section{Genomics}

A Genome Wide Association Study (GWAS) was performed in the discovery cohort and follow-on studies in the replication cohort to identify genetic variants that contribute to the clinical response and outcome of patients included in BIOSTAT-CHF. The GWAS platform was chosen to provide coverage of both common and lower frequency exomic variants. . In addition to identifying independent genetic loci that influence outcome, the findings from the GWAS will be integrated into the development of the general score, the principal aim of BIOSTAT-CHF.

\section{Sample size justification}

The cardiac event rate was expected to be $35 \%$ during the median follow-up of 18 months.(2-5) In addition, dichotomizing the therapy-response variables at six months, we estimated that about $50 \%$ of the patients will respond to their treatment (i.e. patients will not die or have to be hospitalized for 
worsening heart failure). The predictive models were based on between 875-1250 primary events. For the univariable analyses of the associations between SNPs, proteins and biomarkers versus cardiac events the index sample size provides $>80 \%$ power if the odds ratio between a SNP and response/event is 1.4 or if the effect size of a protein/biomarker is 0.12 when using a significance level of 0.05 . But when corrected for the multiple testing on about 500,000 variables the odds ratio and effect size need to be $>2.1$ and 0.26 , respectively, to provide at least $80 \%$ power. It is likely therefore that the index cohort will not identify any definitive hits but will identify a large number of risk markers with suggestive associations with outcomes. These will be taken forward to replication and only those markers showing a combined (discovery + replication) P value will be considered confirmed.

\section{Statistics and Systems biology risk model}

Predictive models for the treatment outcomes will be developed in two ways, using classical statistical methods and by using system biological or computational models based on available knowledge of myocardial adaptation and medication metabolism. The primary outcomes of treatment are all-cause mortality and unplanned hospitalization for heart failure, but we also will consider clinical and functional outcomes on metrics such as NYHA class, left ventricular ejection fraction, blood pressure, quality of life and the six-minute walk test. Development of the predictive models will start with evaluation of univariable associations between the treatment outcome and clinical variables, genetic and biomolecular markers and the protein expressions as assessed at the baseline visit. To limit the familywise error rate due to multiple testing we will lower the overall significance level such that the false discovery rate is between $5 \%$ and $10 \%$. We will use permutations to estimate the false discovery rate (FDR) and the $p$-values. Multiple regression analyses will next be used to select the independent predictors of treatment outcome. We will develop ensembles of different statistical and machinelearning tools such as linear and logistic regression, neural networks, regression trees, random forests and support vector machines. Variable selection will be done with lasso regularization and the optimal lasso-penalties will be determined with cross-validation. Weights for the different regression tools in the ensembles will also be determined with cross-validation. Since the different treatment outcome parameters will be correlated we will also use multivariate regression techniques to develop predictive models: we will in particular use penalized canonical correlation analysis and partial least squares. 
Our second approach to develop predictive models for treatment outcomes will be based on the available measurements of clinical, biomolecular and genetic markers and current knowledge of the pathways involved in myocardial adaptation and the effect of medication/treatment on these pathways. We will use simple causal statistical models for the treatment outcomes by explaining how pathogenic pathways involved in heart failure are modulated by heart failure medication.(Figure 3)

Pathogenetic pathways on development and progression of heart failure will be mined from available knowledge-bases such as NCBI, KEGG, Gene Ontology, and SwissProt. The KEGG database, for example, currently lists several pathways leading to heart failure among which are the pathways for dilated cardiomyopathy (map05414) and hypertrophic cardiomyopathy (map05410). Available genetic and biomolecular markers and proteins that are part of the selected pathways will be modelled using Bayesian network analyses and associated as a whole with the treatment outcomes. In addition, we will use global testing and gene-enrichment approaches to test the involvement of associated pathways such as the renin-angiotensin system, the TGFB-signalling pathway and inflammatory pathways. To predict how the different heart failure medications influence these pathogenetic pathways of heart failure, we will associate these pathways with known metabolic pathways of medications. The metabolic pathways will be mined from NCBI, KEGG, Gene Ontology, PharmGkb and several interaction databases such as Swissprot, Reactomes, Interactome, and BioGrid. Pharmacodynamics and bioavailability of the heart failure medications and their associations with genetic markers will be mined from PharmGkb.

\section{Baseline characteristics of the index and validation cohort}

Baseline characteristics of the patients in the index $(n=2516)$ and validation cohort $(n=1738)$ are presented table 1. Patients in the index cohort were younger and more often male, but overall both cohorts were well matched. The majority of patients were hospitalised for acute heart failure, and the remainder (index cohort: 32.6\%; validation cohort: 46.3\%) presented with worsening signs and/or symptoms of heart failure at the outpatient clinic. At inclusion, $44 / 48 \%$ of patients were in NYHA class III and $10-15 \%$ of patients had HFpEF. According to study design, all patients used diuretics, but due to the inclusion criteria of both cohorts, patients were not on optimal, evidence-based medical therapy: 70/72\% used an ACE-inhibitor/Angiotensin Receptor Blocker, and < 50\% used the optimal dose. Betablockers were used in $73 / 83 \%$ of patients and only $32 / 46 \%$ used the optimal dose. 


\section{Discussion}

From the 1980s to now, the outcome of heart failure patients has tremendously improved. The introduction of ACE-inhibitors, beta-blockers, angiotensin receptor blockers and mineralocorticoid receptor antagonists have resulted in symptom improvement and a lower risk for mortality and hospital admission for worsening heart failure. Medical devices, such as ICDs and cardiac resynchronization therapy further improved outcomes. Therefore, it will be more difficult to make further progression in the treatment of patients with heart failure. Given the large heterogeneity of patients with heart failure, a one-size-fits-all approach is likely not lead to major further improvements. We suggest that future significant improvements can only be achieved when therapy is individualized and tailored to the patient's profile.

At present, individualized medicine is already carefully applied in patients with heart failure. Cardiac resynchronization therapy is only recommended in patients with a QRS width $>130$ ms and patients with a left bundle branch block seem to respond better.(3) Ivabradine is only recommended in patients with a higher heart rate and are in sinus rhythm.(3) Recent and ongoing trials in acute heart failure are aimed at patients with a systolic blood pressure $>125 \mathrm{mmHg}$. $(11,12)$ However, these examples are based on the mode of action of the drug, and is only aimed at major and expected response profiles.

To detect unexpected response profiles, unbiased approached are needed. Multiple large phase III randomized clinical trials provide pre-defined subgroup analyses, which are usually presented by Forest plots. However, these conventional subgroup analyses based on clinical characteristics often failed to demonstrate any interaction effects with treatment. One exception is that African-American heart failure patients seem to respond less well to ACE-inhibitors. (13) Similar findings were observed on the response of African American patients to angiotensin receptor blockers. A few other examples of patient characteristics that influence the response to therapy have been described in a recent review.(14) Biomarkers have become increasingly important in evaluating the diagnosis, prognosis and phenotype of patients with heart failure(15-18), but they might also predict the response to therapy. For example, in patients with chronic heart failure and elevated renin levels, ACE-inhibitors cause a marked diuresis, which is not observed in patients with low renin levels.(19) Similarly, the blood pressure lowering effects of several groups of anti-hypertensive drugs is highly associated with plasma renin activity.(20) Interestingly, the less pronounced response of African American patients to ACE inhibition might be due to their lower renin activity.(21) Several genetic variations have also been related to the response to heart failure therapy. For example, patients with the Arg389Arg genotype showed a marked 
improvement in survival and reduction in hospitalization on the beta-blocker bucindolol, while only a minimal impact was evident in patients either homozygous or heterozygous for the Gly389 allele.(22) Other examples of genes influencing the response to therapy are depicted in figure 2 and reviewed elsewhere.(14)

In summary, the individualized response to a drug can be studied on different levels, such as on a genetic, proteomic, and phenotypic level. However, as depicted in figure 1, these levels are internally related to each other. For example, there are several genetic variations that are related to the activity of the renin-angiotensin system, which define several phenotypes of patients with a better or worse response to inhibitors of the renin-angiotensin system.(Figure 2)

BIOSTAT-CHF aims to integrate demographics, established biomarkers, proteomic, and genetic information to achieve a better assessment of the individual patient's characteristics, response to therapy and prognosis. This necessitates either the discovery of new markers and/or their integration with known variables into a systems biology approach, which may identify groups of patients that have a poor response to the current recommended therapy for heart failure.

Systems biology focuses on the complex interactions in biological systems from a new perspective; integration instead of reduction.(23) This approach requires the integration of experimental and computational research to help understand a system's structure and dynamics. Multilevel data from BIOSTAT-CHF will be analyzed using logistic regression models, but also using alternatives, such as logic regression, neural networks, regression trees, random forests and support vector machines, to determine characteristics of patients that are associated with the response to current heart failure treatment. Taken together, using a systems biology approach, BIOSTAT-CHF aims to detect pathways which may explain the individual patient response to therapy.

BIOSTAT-CHF successfully recruited an index cohort $(n=2516)$ and a validation cohort $(n-1738)$ of patients with worsening signs and/or symptoms of heart failure who were not yet optimally treated. These populations are at high risk for subsequent events, such as mortality and/or heart failure (re)hospitalization. Due to the stricter (NT-pro)BNP criteria for patients with a LVEF $>40 \$$, only $10-15 \%$ of the patients has HFpEF. Both cohorts were well comparable in terms of their characteristics and both had a median follow-up of 21 months. 


\section{Summary}

Although major improvements have been achieved in the treatment of patients with heart failure, mortality and morbidity remain high. The large variation in patient profiles, aetiology, cardiac phenotypes, co-morbidities and clinical presentation led to the hypothesis that not all patients will benefit from the same treatment. Therefore, we believe that the next step forward has to come from an individualized treatment approach. In BIOSTAT-CHF, two independent cohorts $(n=2516$ and $n=1738$ ) of worsening heart failure patients were recruited, their medication was optimized, and patients were followed for a median of 21 months. By using a novel systems biology approach we will provide a model that predicts response to therapy, incorporating demographics, biomarkers, genome-wide analysis, and proteomics. Such a predictive model should be instrumental in developing alternative therapies for patients with a sub-optimal response and thus improve care for patients with heart failure.

Funding: This project was funded by a grant from the European Commission: FP7-242209-BIOSTAT-CHF

\section{Conflicts of Interest:}

Voors reports consultancy fees and/or research grants from: Alere, Amgen, Bayer, Boehringer Ingelheim, Cardio3Biosciences, Celladon, GSK, Merck/MSD, Novartis, Servier, Stealth Peptides, Singulex, Sphingotec, Trevena, Vifor, ZS Pharma

Anker reports grants from Vifor and Abbott Vascular, and fees for consultancy from Vifor, Bayer, Boehringer Ingelheim, Brahms, Cardiorentis, Janssen, Novartis, Relypsa, Servier, Stealth Peptides and ZS Pharma.

Cleland:

Dickstein:

Filippatos: received fees and/ or research grants from Novartis, Bayer, Cardiorentis, Vifor, Servier, Alere, Abbott

Van der Harst: research grant from Abbott. 
Hillege:

Lang: received consultancy fees and/or research grants from: Amgen, Astra Zeneca, MSD, Novartis, Servier

$\mathrm{Ng}$ : none

Ponikowski:

Samani: None

Van Veldhuisen reports Board Membership fees/Travel Expenses from BioControl, Cardiorentis, Johnson \& Johnson, Novartis, Vifor and Zoll Medical.

Zannad:

Zwinderman:

Metra: Consulting honoraria from Bayer, Novartis, Servier, speaker's fees from Abbott vascular, Bayer and ResMed.

\section{List of collaborators:}

WP1: Project Management team: A.A. Voors (WP-leader), S.D. Anker, J.G. Cleland, K. Dickstein, G. Filippatos, H.L. Hillege, C.C. Lang, MD, M. Metra, L. Ng, P. Ponikowski, N. Samani, D.J. van Veldhuisen, F. Zannad, A.H. Zwinderman.

WP2: Protocols: M. Metra (WP-leader)

WP3: Biomarkers: S. D. Anker (WP-leader)

WP4: Genomics: N. J. Samani (WP-leader), Andrea Koekemoer (Leicester), Manolo Papakonstantinou (Leicester), Leanne M Hall (Leicester), Simon R Romaine (Leicester), Christopher P Romaine (Leicester), 
John R Thompson (Leicester), Pim van der Harst (Groningen)WP5: Proteomics: L. Ng (WP-leader): D.J.L. Jones, R. Willingale, H.T. Cao, J.K. Sandhu, P.A. Quinn, H. Patel, J. Auluck, A. Hakimi

WP6: Clinical Study: H.L. Hillege (WP-leader)

WP7: Systems Biology: A.H. Zwinderman (WP-leader)

WP8: Validation Study: C.C. Lang (WP-leader) TAYSIDE: Prof C C Lang, Prof A D Srtuthers, Dr A M Choy, Dr A Doney, Prof C Palmer, Prof A Morris, Prof B Guthrie, Dr H Parry, Dr R Tavendale, Duncan Heather, Lynn Rutherford, Helen Waldie, Mohanpradeep Mohan, Fatima Baig, Pippa Hopkinson, Daniel Levin FIFE HOSPITALS: Dr Mark Francis, Valerie Bryson, ABERDEEN ROYAL INFIRMARY: Dr Dana Dawson, Professor Michael Frenneaux; EDINBURGH ROYAL INFIRMARY: Dr Martin Denvir, Laura Flint, Shirley Robertson; GLASGOW GOLDEN JUBILEE HOSPITAL: Dr Roy Gardner, Marion McAdam, Kirsty McGovern; GLASGOW WESTERN INFIRMARY: Prof J McMurray, Dr Ross Campbell, Dr Jane Cannon 
Table 1. Baseline characteristics of the Index and validation cohort of BIOSTAT-CHF

\begin{tabular}{|c|c|c|c|}
\hline Variable & & Index cohort & Validation cohort \\
\hline $\mathrm{N}=$ & & 2516 & 1738 \\
\hline \multicolumn{4}{|l|}{ Demographics } \\
\hline Sex (\% Male(n)) & & $73.4(1846)$ & $65.9(1145)$ \\
\hline Age (years) & & $68.9 \pm 12$ & $73.7 \pm 10.7$ \\
\hline Race (\% Caucasian(n)) & & $98.9(2489)$ & $99.3(1725)$ \\
\hline $\mathrm{BMI}(\mathrm{kg} / \mathrm{m} 2)$ & & $27.9 \pm 5.5$ & $28.1 \pm 6.4$ \\
\hline Weight (kg) & & $81.8 \pm 18.5$ & $82.2 \pm 20.1$ \\
\hline Height $(\mathrm{cm})$ & & $170.9 \pm 9.2$ & $168 \pm 10.4$ \\
\hline \multicolumn{4}{|l|}{ NYHA class $(\%(n))$} \\
\hline & $\mathrm{I}$ & $2.2(56)$ & $1.0(17)$ \\
\hline & II & $34.5(868)$ & $41.0(712)$ \\
\hline & III & $48.8(1228)$ & $44.4(772)$ \\
\hline & IV & $11.7(294)$ & $13.6(236)$ \\
\hline \multirow[t]{2}{*}{$\overline{\operatorname{LVEF}(\%)}$} & & $31 \pm 10.6$ & $16 \%$ Good, $79 \%$ \\
\hline & & & Poor/Reduced 5\% N/A \\
\hline \multicolumn{4}{|l|}{ Clinical Profile } \\
\hline Edema $(\%(n))$ & & $29.7(624)$ & $54.9(955)$ \\
\hline Orthopnea (\%(n)) & & $35(879)$ & $4.1(71)$ \\
\hline Rales $>1 / 3$ up lung fiels $(\%(n))$ & & $19.2(248)$ & $2.9(50)$ \\
\hline Jugular venous pressure $(\%(n))$ & & $33.5(554)$ & $25.9(450)$ \\
\hline Hepatomegaly (\%(n)) & & $14.3(358)$ & $3.5(60)$ \\
\hline Systolic Blood Pressure (mmHg) & & $124.7 \pm 21.9$ & $125.9 \pm 22.6$ \\
\hline
\end{tabular}




\begin{tabular}{|c|c|c|}
\hline Diastolic Blood Pressure (mmHg) & $74.9 \pm 13.4$ & $69.2 \pm 13.2$ \\
\hline Heart Rate (beats/min) & $80 \pm 19.5$ & $74.2 \pm 16.6$ \\
\hline \multicolumn{3}{|l|}{ Hospitalization } \\
\hline \multicolumn{3}{|l|}{ Type of visit (\%(n)) } \\
\hline Scheduled outpatient clinic & $27.2(685)$ & $46.3(804)$ \\
\hline Unscheduled outpatient clinic & $5.4(137)$ & $0(0)$ \\
\hline Inpatient hospitalization & $67.3(1694)$ & $53.7(934)$ \\
\hline \multicolumn{3}{|l|}{ Reason for visit (\%(n)) } \\
\hline Worsening heart failure & $54.5(1372)$ & $76.9(1323)$ \\
\hline New onset heart failure & $27.9(702)$ & $23.9(415)$ \\
\hline Other & $17.6(442)$ & $0(0)$ \\
\hline \multicolumn{3}{|l|}{ Heart Failure History } \\
\hline Years since first diagnosis & $2.8[0.1-9.4]$ & $1.4(0.1-4.7)$ \\
\hline Ischemic heart disease $(\%(n))$ & $60.5(1358)$ & $64.9(1128)$ \\
\hline Previous HF hospitalization $(\%(n))$ & $31.6(794)$ & $26.5(460)$ \\
\hline \multicolumn{3}{|l|}{ Medical History } \\
\hline Hypertension (\%(n)) & $62.4(1569)$ & $57.9(1007)$ \\
\hline Atrial fibrillation $(\%(n))$ & $45.4(1143)$ & $43.7(760)$ \\
\hline Myocardial infarction (\%(n)) & $38.3(963)$ & $48.8(849)$ \\
\hline $\mathrm{PCl}(\%(n))$ & $21.6(544)$ & $18.7(325)$ \\
\hline CABG $(\%(n))$ & $17.2(433)$ & $17.7(308)$ \\
\hline Pacemaker (\%(n)) & $7.3(183)$ & $6.6(115)$ \\
\hline $\operatorname{ICD}(\%(n))$ & $8.1(205)$ & $4.0(69)$ \\
\hline Biventricular pacer (CRT) (\%(n)) & $1.9(49)$ & $1.6(27)$ \\
\hline
\end{tabular}




\begin{tabular}{|c|c|c|}
\hline Biventricular Pacer (CRT(n)) and ICD (\%(n)) & $6.9(173)$ & $3.2(55)$ \\
\hline Diabetes mellitus (\%(n)) & $32.6(819)$ & $32.3(561)$ \\
\hline COPD (\%(n)) & $17.3(436)$ & $18.4(319)$ \\
\hline Peripheral artery disease $(\%(n))$ & $10.9(273)$ & $21.5(374)$ \\
\hline Stroke $(\%(n))$ & $9.3(233)$ & $18.1(315)$ \\
\hline \multicolumn{3}{|l|}{ Medication } \\
\hline $\begin{array}{l}\text { ACE-inhibitors or Angiotensin receptor } \\
\text { blockers }(\%(n))\end{array}$ & $72.3(1820)$ & $70.1(1218)$ \\
\hline Target dose $(\%(n))$ & $13.4(336)$ & $15.2(185)$ \\
\hline$\%$ of optimal dose & $48 \pm 35$ & $43 \pm 27$ \\
\hline Beta-blockers (\%(n)) & $83.2(2093)$ & $72.7(1264)$ \\
\hline Target dose $(\%(n))$ & $5.5(138)$ & $17.8(226)$ \\
\hline$\%$ of optimal dose & $32 \pm 26$ & $46 \pm 28$ \\
\hline Loop diuretics $(\%(\mathrm{n}))$ & $99.5(2504)$ & $99.4(1728)$ \\
\hline Aldosterone antagonists (\%(n)) & $53.2(1339)$ & $32.5(564)$ \\
\hline Digoxine $(\%(n))$ & $19.5(491)$ & $17.9(311)$ \\
\hline \multicolumn{3}{|l|}{ Laboratory } \\
\hline Hemoglobin (g/dL) & $13.3[11.9-14.5]$ & $13.2(11.8-14.5)$ \\
\hline Creatinine (umol/L) & $101.0[82.6-128.2]$ & $97.0(79.8-125.0)$ \\
\hline Urea (mmol/L) & $11.1[7.4-17.7]$ & $8.6(6.5-11.9)$ \\
\hline GFR MDRD formula (ml/min/1.73m²) & $67.1[50.4-86.1]$ & $63.3(46.9-80.4)$ \\
\hline Sodium (mmol/L) & $140.0[137.0-142.0]$ & $139.0(137.0-141.0)$ \\
\hline Potassium (mmol/L) & $4.2[3.9-4.6]$ & $4.3(4.0-4.6)$ \\
\hline $\mathrm{BNP}(\mathrm{pg} / \mathrm{mL})$ & $667.8[362-1266.5]$ & \\
\hline
\end{tabular}


Table 2. Overview of biomarkers and associated pathophysiological domains

\begin{tabular}{|c|c|c|c|c|c|c|c|c|c|c|c|c|}
\hline & 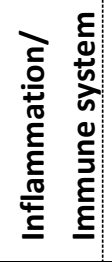 & 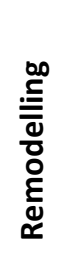 & 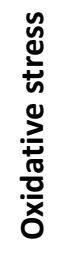 & 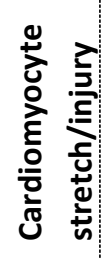 & 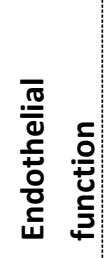 & 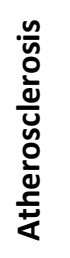 & 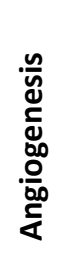 & $\frac{5}{\frac{0}{2}}$ & 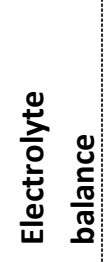 & 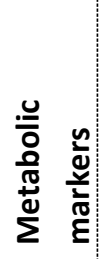 & 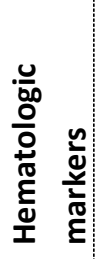 & 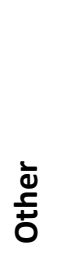 \\
\hline Albumin & $x$ & & & & & & & & & $x$ & & $x$ \\
\hline Aldosterone & & & & & & & & & & & & $x$ \\
\hline ALT & & & & & & & & & & & & $x$ \\
\hline Alkaline phosphatase & & & & & & & & & & & & $x$ \\
\hline ANP & & & & $\mathbf{x}$ & & & & & & & & \\
\hline AST & & & & & & & & & & & & $x$ \\
\hline Angiogenin & & & & & & & $x$ & & & & & \\
\hline BUN & & & & & & & & $x$ & & $x$ & & \\
\hline (NT-pro) BNP & & & & $x$ & & & & & & & & \\
\hline Calcium & & & & & & & & & $x$ & & & $x$ \\
\hline Creatinine & & & & & & & & $x$ & & & & \\
\hline CRP & $x$ & & & & & $x$ & & & & & & \\
\hline Cystatin C & $x$ & & & & & & & $x$ & & & & \\
\hline D-Dimer & $x$ & & & & & & & & & & & $x$ \\
\hline Endothlin-1 & & & & & $x$ & & & & & & & \\
\hline ESAM & $x$ & & & & & $x$ & $x$ & & & & & \\
\hline Erythrocytes & & & & & & & & & & & $x$ & \\
\hline
\end{tabular}




\begin{tabular}{|c|c|c|c|c|c|c|c|c|c|c|c|c|}
\hline & 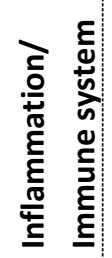 & 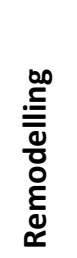 & 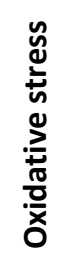 & 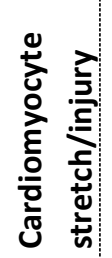 & 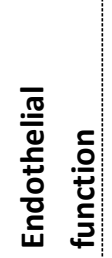 & 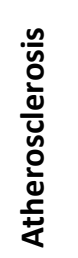 & 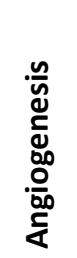 & 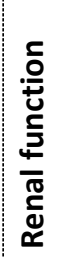 & 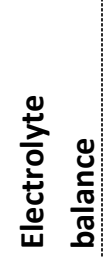 & 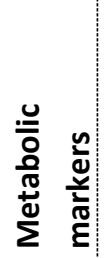 & 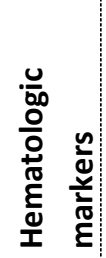 & 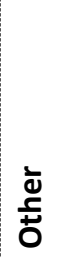 \\
\hline Ferritin & $x$ & & & & & & & & & & $x$ & \\
\hline FGF-23 & & & & & & & & & & & & $x$ \\
\hline Galectin-3 & $x$ & $x$ & & & & & & $x$ & & & & \\
\hline Gamma GT & & & & & & & & & & & & $x$ \\
\hline GDF-15 & $x$ & $x$ & $x$ & & & $x$ & & & & & & \\
\hline Glucose & & & & & & & & & & $x$ & & \\
\hline HDL & & & & & & & & & & $x$ & & \\
\hline Hemoglobin & & & & & & & & & & & $x$ & \\
\hline Hematocrit & & & & & & & & & & & $x$ & \\
\hline Hepcidin & & & & & & & & & & & $x$ & \\
\hline Interleukin-6 & $x$ & & & & & & & & & & & \\
\hline Iron & & & & & & & & & & & $x$ & \\
\hline LDL & & & & & & $x$ & & & & $x$ & & \\
\hline Leucocytes & $x$ & & & & & & & & & & $x$ & \\
\hline LTßR & $x$ & & & & & $x$ & & & & & & \\
\hline Mesothelin & & & & & & & & & & & & $x$ \\
\hline MR-proADM & & & & & $x$ & & & & & & & \\
\hline Myeloperoxidase & $x$ & & $x$ & & & & & & & & & \\
\hline
\end{tabular}




\begin{tabular}{|c|c|c|c|c|c|c|c|c|c|c|c|c|}
\hline & 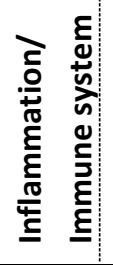 & 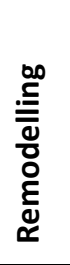 & 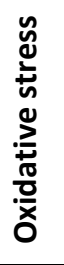 & 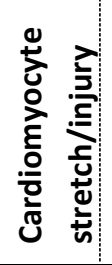 & 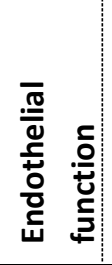 & 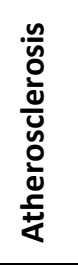 & 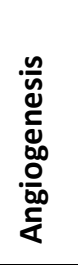 & $\frac{5}{0}$ & 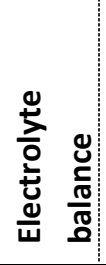 & 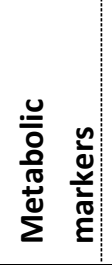 & 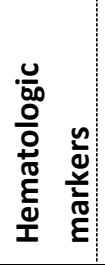 & 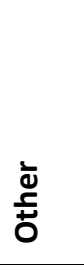 \\
\hline Neuropilin & & & & & $x$ & & $x$ & & & & & $x$ \\
\hline NGAL & $x$ & & & & & & & $x$ & & & & \\
\hline NT-proCNP & & & & & $x$ & & & & & & & \\
\hline Osteopontin & $x$ & $x$ & & & & $x$ & $x$ & & & & & \\
\hline Procalcitonin & $x$ & & & & & & & & & & & \\
\hline Pentraxin-3 & $x$ & & & & & & & & & & & \\
\hline Periostin & & $x$ & & & & & & & & & & $x$ \\
\hline Phosphate & & & & & & & & & $x$ & & & $x$ \\
\hline PIGR & $x$ & & & & & & & & & & & \\
\hline Platelets & & & & & & & & & & & $x$ & $x$ \\
\hline Potassium & & & & & & & & $x$ & $x$ & & & \\
\hline Pro-enkephalin & & & & & & & & & & & & $x$ \\
\hline PSAP-B & & & $x$ & & & & & & & & & $x$ \\
\hline RAGE & $x$ & & & & & $x$ & & & & & & \\
\hline Renin & & & & & & & & & & & & $x$ \\
\hline sST-2 & $x$ & $x$ & $x$ & $x$ & & & $x$ & & & & & \\
\hline Sodium & & & & & & & & $x$ & $x$ & & & \\
\hline $\begin{array}{l}\text { Soluble transferrin } \\
\text { receptor }\end{array}$ & & & & & & & & & & & $x$ & \\
\hline
\end{tabular}




\begin{tabular}{|c|c|c|c|c|c|c|c|c|c|c|c|c|}
\hline & 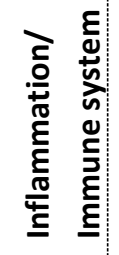 & 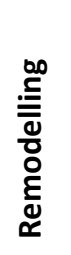 & 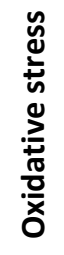 & 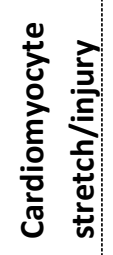 & 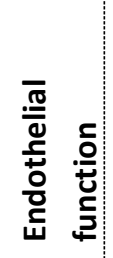 & 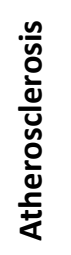 & 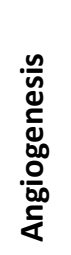 & 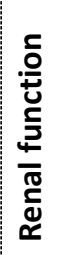 & 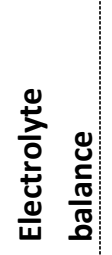 & 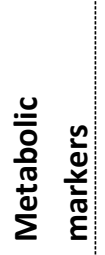 & 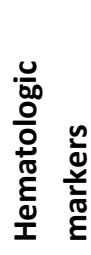 & 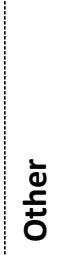 \\
\hline Syndecan-1 & $x$ & $\mathbf{x}$ & & & & & & & & & & \\
\hline TNFR-1 & $x$ & & & & & & & & & & & \\
\hline Total bilirubin & & & & & & & & & & & $x$ & \\
\hline Total cholesterol & & & & & & $x$ & & & & $\mathbf{x}$ & & \\
\hline Transferrin & & & & & & & & & & & $x$ & \\
\hline Triglycerides & & & & & & $x$ & & & & $x$ & & \\
\hline Troponin & & & & $x$ & & & & & & & & \\
\hline Troy & $x$ & $x$ & & & & & & & & & & \\
\hline VEGFR-1 & & & & & & & $x$ & & & & & \\
\hline WAP-4C & $x$ & & & & & & & & & & & $x$ \\
\hline
\end{tabular}


Figure 1. Schematic overview of the BIOSTAT-CHF project. WP= Work Package.

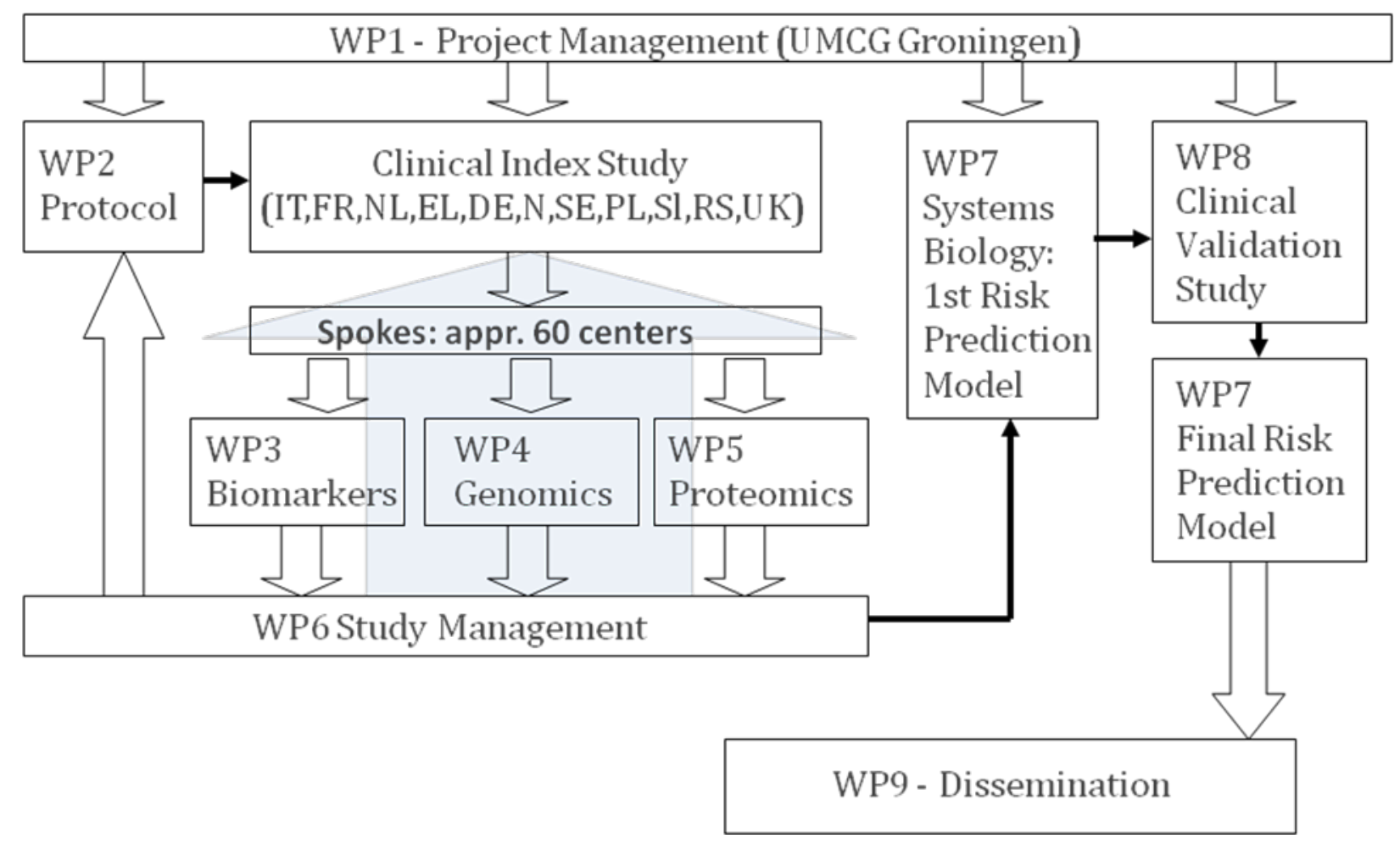


Figure 2. Example of a genetic profile, proteins and phenotypes related to the activity of the reninangiotensin aldosterone system that might influence the response to blockers of the renin-angiotensin aldosterone system.

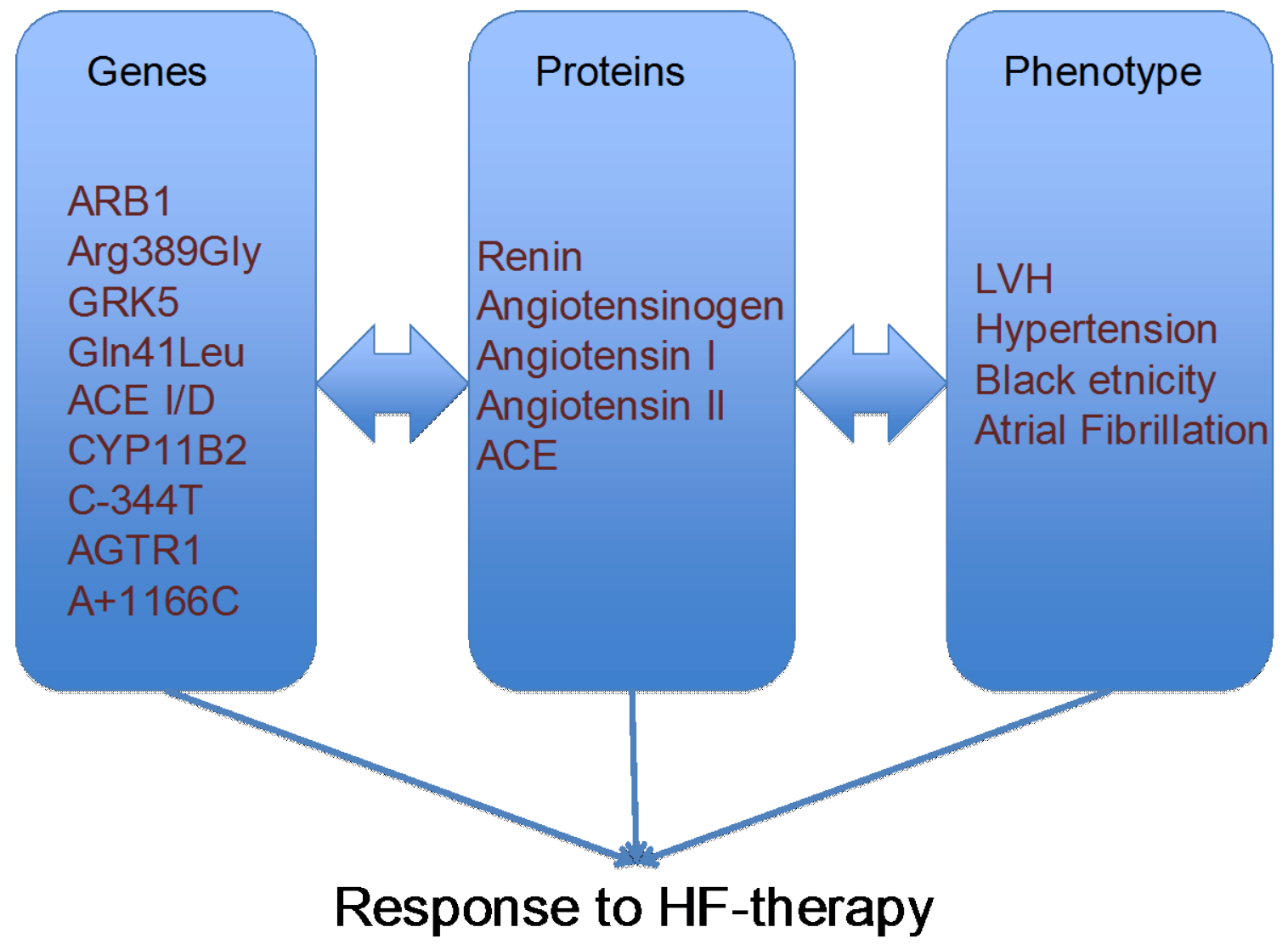


Figure 3. Rationale and design of BIOSTAT-CHF: to use a system biology approach integrating genetic, protein, and clinical information in order to define a responder profile to current recommended therapy for patients with heart failure. $A C E i=a n g i o t e n s i n$ converting enzyme-inhibitor; $A R B=$ angiotensin receptor blocker; $B B=$ beta blocker; GWAS + genome wide association study; $M R A=$ mineralocorticoid receptor antagonist; $W H F=$ worsening heart failure

\section{Index Cohort:}

$-\mathrm{N}=2516$ WHF patients

-12 European Countries

-Samples: GWAS/proteomics/biomarkers -ACEi/ARB+BB+MRA uptitrated -Follow-up $\geq 18$ months
Validation Cohort:

$-\mathrm{N}=1738$ WHF patients

-Scotland

-Samples: GWAS/proteomics/biomarkers

-ACEi/ARB+BB+MRA uptitrated

-Follow-up $\geq 6$-36 months

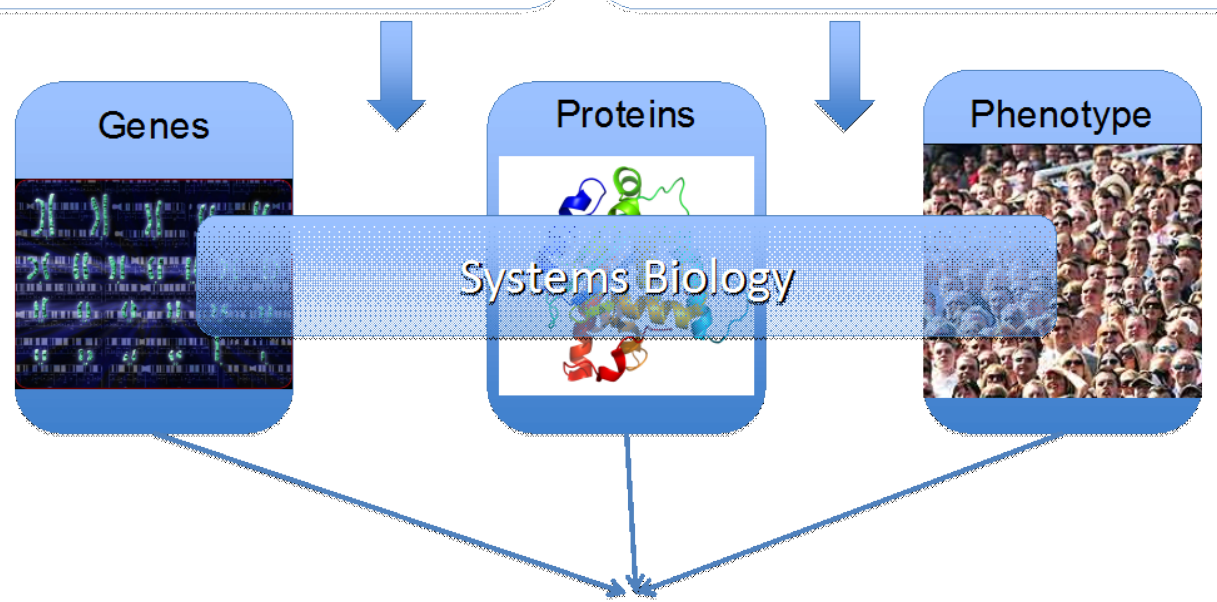

"Response to therapy Risk Score" (death or HF-hospitalization) 


\section{References}

(1) Mosterd A, Hoes AW. Clinical epidemiology of heart failure. Heart 2007 Sep;93(9):1137-1146.

(2) Tavazzi L, Senni M, Metra M, Gorini M, Cacciatore G, Chinaglia A, et al. Multicenter prospective observational study on acute and chronic heart failure: one-year follow-up results of IN-HF (Italian Network on Heart Failure) outcome registry. Circ Heart Fail 2013 May;6(3):473-481.

(3) McMurray JJ, Adamopoulos S, Anker SD, Auricchio A, Bohm M, Dickstein K, et al. ESC guidelines for the diagnosis and treatment of acute and chronic heart failure 2012: The Task Force for the Diagnosis and Treatment of Acute and Chronic Heart Failure 2012 of the European Society of Cardiology. Developed in collaboration with the Heart Failure Association (HFA) of the ESC. Eur J Heart Fail 2012 Aug;14(8):803-869.

(4) Mebazaa A, Yilmaz MB, Levy P, Ponikowski P, Peacock WF, Laribi S, Ristic AD, Lambrinou E, Masip J, Riley JP, McDonagh T, Mueller C, deFilippi C, Harjola VP, Thiele H, Piepoli MF, Metra M, Maggioni A, McMurray J, Dickstein K, Damman K, Seferovic PM, Ruschitzka F, Leite-Moreira AF, Bellou A, Anker SD, Filippatos G. Recommendations on pre-hospital \& early hospital management of acute heart failure: a consensus paper from the Heart Failure Association of the European Society of Cardiology, the European Society of Emergency Medicine and the Society of Academic Emergency Medicine. Eur J Heart Fail. 2015 Jun;17(6):544-58.

(5) Maggioni AP, Dahlstrom U, Filippatos G, Chioncel O, Crespo Leiro M, Drozdz J, et al. EURObservational Research Programme: regional differences and 1-year follow-up results of the Heart Failure Pilot Survey (ESC-HF Pilot). Eur J Heart Fail 2013 Jul;15(7):808-817.

(6) McMurray JJ. CONSENSUS to EMPHASIS: the overwhelming evidence which makes blockade of the renin-angiotensin-aldosterone system the cornerstone of therapy for systolic heart failure. Eur J Heart Fail 2011 Sep;13(9):929-936.

(7) Fox K, Ford I, Steg PG, Tendera M, Ferrari R, BEAUTIFUL Investigators. Ivabradine for patients with stable coronary artery disease and left-ventricular systolic dysfunction (BEAUTIFUL): a randomised, double-blind, placebo-controlled trial. Lancet 2008 Sep 6;372(9641):807-816.

(8) Kjekshus J, Apetrei E, Barrios V, Bohm M, Cleland JG, Cornel JH, et al. Rosuvastatin in older patients with systolic heart failure. N Engl J Med 2007 Nov 29;357(22):2248-2261.

(9) Konstam MA, Gheorghiade M, Burnett JC,Jr, Grinfeld L, Maggioni AP, Swedberg K, et al. Effects of oral tolvaptan in patients hospitalized for worsening heart failure: the EVEREST Outcome Trial. JAMA 2007 Mar 28;297(12):1319-1331.

(10) Dickstein K, Cohen-Solal A, Filippatos G, McMurray JJ, Ponikowski P, Poole-Wilson PA, Strömberg A, van Veldhuisen DJ, Atar D, Hoes AW, Keren A, Mebazaa A, Nieminen M, Priori SG, Swedberg K; ESC Committee for Practice Guidelines (CPG). ESC guidelines for the diagnosis and treatment of acute and chronic heart failure 2008: the Task Force for the diagnosis and treatment of acute and chronic heart failure 2008 of the European Society of Cardiology. Developed in collaboration with the Heart Failure 
Association of the ESC (HFA) and endorsed by the European Society of Intensive Care Medicine (ESICM). Eur J Heart Fail. 2008 Oct;10(10):933-89.

(11) Levy PD, Laribi S, Mebazaa A. Vasodilators in Acute Heart Failure: Review of the Latest Studies. Curr Emerg Hosp Med Rep. 2014 Jun;2(2):126-132.

(12) Mebazaa A, Longrois D, Metra M, Mueller C, Richards AM, Roessig L, Seronde MF, Sato N, Stockbridge NL, Gattis Stough W, Alonso A, Cody RJ, Cook Bruns N, Gheorghiade M, Holzmeister J, Laribi $\mathrm{S}$, Zannad F. Agents with vasodilator properties in acute heart failure: how to design successful trials. Eur J Heart Fail. 2015 Jul;17(7):652-64

(13) Exner DV, Dries DL, Domanski MJ, Cohn JN. Lesser response to angiotensin-converting-enzyme inhibitor therapy in black as compared with white patients with left ventricular dysfunction. N Engl J Med 2001 May 3;344(18):1351-1357.

(14) Liu LC, Voors AA, Valente MA, van der Meer P. A novel approach to drug development in heart failure: towards personalized medicine. Can J Cardiol 2014 Mar;30(3):288-295.

(15) Schmitter D, Cotter G, Voors AA. Clinical use of novel biomarkers in heart failure: towards personalized medicine. Heart Fail Rev 2014 May;19(3):369-381.

(16) de Boer RA, Daniels LB, Maisel AS, Januzzi JL Jr. State of the Art: Newer biomarkers in heart failure. Eur J Heart Fail. 2015 Jun;17(6):559-69

(17) Richards AM, Frampton CM. Can circulating biomarkers identify heart failure patients at low risk? Eur J Heart Fail. 2015 doi: 10.1002/ejhf.438. [Epub ahead of print] No abstract available. PMID: 26537440

(18) Sanders-van Wijk S, van Empel V, Davarzani N, Maeder MT, Handschin R, Pfisterer ME, Brunner-La Rocca HP; TIME-CHF investigators. Circulating biomarkers of distinct pathophysiological pathways in heart failure with preserved vs. reduced left ventricular ejection fraction. Eur J Heart Fail. 2015 Oct;17(10):1006-14.

(19) Lim PO, MacFadyen RJ, Struthers AD. Is there a role for renin profiling in selecting chronic heart failure patients for ACE inhibitor treatment? Heart 2000 Mar;83(3):257-261.

(20) Preston RA, Materson BJ, Reda DJ, Williams DW, Hamburger RJ, Cushman WC, et al. Age-race subgroup compared with renin profile as predictors of blood pressure response to antihypertensive therapy. Department of Veterans Affairs Cooperative Study Group on Antihypertensive Agents. JAMA 1998 Oct 7;280(13):1168-1172.

(21) He J, Klag MJ, Appel LJ, Charleston J, Whelton PK. The renin-angiotensin system and blood pressure: differences between blacks and whites. Am J Hypertens 1999 Jun;12(6):555-562.

(22) Liggett SB, Mialet-Perez J, Thaneemit-Chen S, Weber SA, Greene SM, Hodne D, et al. A polymorphism within a conserved beta(1)-adrenergic receptor motif alters cardiac function and betablocker response in human heart failure. Proc Natl Acad Sci U S A 2006 Jul 25;103(30):11288-11293. 
(23) Kitano H. Computational systems biology. Nature 2002 Nov 14;420(6912):206-210. 